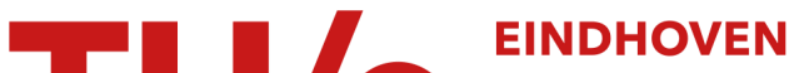 UNIVERSITY OF TECHNOLOGY
}

\section{Effects of vibration-induced fatigue on the H-reflex}

Citation for published version (APA):

Sammali, F., Xu, L., Rabotti, C., Cardinale, M., Xu, Y., van Dijk, J. P., Zwarts, M. J., Del Prete, Z., \& Mischi, M. (2018). Effects of vibration-induced fatigue on the H-reflex. Journal of Electromyography and Kinesiology, 39, 134-141. https://doi.org/10.1016/j.jelekin.2018.02.005

DOI:

10.1016/j.jelekin.2018.02.005

Document status and date:

Published: 01/04/2018

\section{Document Version:}

Accepted manuscript including changes made at the peer-review stage

\section{Please check the document version of this publication:}

- A submitted manuscript is the version of the article upon submission and before peer-review. There can be important differences between the submitted version and the official published version of record. People interested in the research are advised to contact the author for the final version of the publication, or visit the $\mathrm{DOI}$ to the publisher's website.

- The final author version and the galley proof are versions of the publication after peer review.

- The final published version features the final layout of the paper including the volume, issue and page numbers.

Link to publication

\section{General rights}

Copyright and moral rights for the publications made accessible in the public portal are retained by the authors and/or other copyright owners and it is a condition of accessing publications that users recognise and abide by the legal requirements associated with these rights.

- Users may download and print one copy of any publication from the public portal for the purpose of private study or research.

- You may not further distribute the material or use it for any profit-making activity or commercial gain

- You may freely distribute the URL identifying the publication in the public portal.

If the publication is distributed under the terms of Article $25 \mathrm{fa}$ of the Dutch Copyright Act, indicated by the "Taverne" license above, please follow below link for the End User Agreement:

www.tue.nl/taverne

\section{Take down policy}

If you believe that this document breaches copyright please contact us at:

openaccess@tue.nl

providing details and we will investigate your claim. 


\title{
Effects of vibration-induced fatigue on the H-reflex
}

\author{
F. Sammali ${ }^{a}$, L. Xu ${ }^{a, *}$, C. Rabotti ${ }^{a}$, M. Cardinale ${ }^{b, c, d}$, Y. Xu $^{a}$, J.P. van \\ Dijk $^{e}$, M.J. Zwarts ${ }^{e}$, Z. Del Prete ${ }^{f}$, and M. Mischi ${ }^{a}$ \\ ${ }^{a}$ Eindhoven University of Technology, Eindhoven, the Netherlands \\ ${ }^{b}$ Aspire academy, Doha, Qatar \\ ${ }^{c}$ University College London, London, UK \\ ${ }^{d}$ University of St. Mark and St. John, Plymouth, UK \\ ${ }^{e}$ Kempenhaeghe Academic Centrum for Epilepsy, Heeze, the Netherlands \\ $f$ 'La Sapienza' University of Rome, Rome, Italy
}

\begin{abstract}
Vibration exercise (VE) has been suggested as an effective training for improving muscle strength and coordination. However, the underlying physiological adaptation processes are not yet fully understood, limiting the development of safe and effective exercise protocols. To better understand the neuromuscular responses elicited by VE, we aimed at investigating the acute effects of superimposed vibration on the Hoffmann reflex (H-reflex), measured after fatiguing exercise. Twenty-five volunteers performed four isometric contractions of the right Flexor Carpi Radialis (FCR) with baseline load at $80 \%$ of their maximal voluntary contraction (MVC), both with no vibration and with superimposed vibration at 15, 30, and $45 \mathrm{~Hz}$. Fatigue was estimated by MVC test and estimation of electromyographic spectral compression. Hreflex suppression was estimated as the relative decrease after exercise. Our results show that fatiguing exercise determined a decrease in H-reflex amplitude compared to rest condition while vibration determined a lower H-reflex suppression as compared to no vibration. The superimposition of $30-\mathrm{Hz}$ vibration determined the largest acute reduction in force generating capacity $(36 \mathrm{~N}, p<0.05)$ and the lowest H-reflex suppression $(20 \%, p<0.05)$. These results suggest VE to be particularly suitable in rehabilitation programs for rapid restoration of muscle form and function after immobilization periods.
\end{abstract}

Keywords: Electromyography, Vibration exercise, H-reflex, Tonic vibration reflex, Maximal voluntary contraction, Fatigue 


\section{Introduction}

Resistance exercise and physical training have always been considered the most effective options for improving, maintaining or restoring skeletal muscle form and function [1]. Improvements in muscle form and function are usually sought using conventional training methods such as resistance exercise and plyometrics [2, 3] or less conventional methods like electrical stimulation [4] and blood flow restriction [5]. These training methods have been shown to determine improvements in neuromuscular performance as measured by force increase and mechanical power production in various populations and age groups.

Over the last decade, an alternative form of exercise, characterized by the use of vibration with oscillating platforms $[6,7,8]$, has been suggested as an effective methodology for improving strength and power performance $[9,10]$ mostly in non athletic populations. Whole body vibration as an exercise modality has recently become a topic of great interest, and recent research has focused on its potential benefits. Vibration exercise (VE) seems to elicit a specific warm-up effect, improving muscle power and balance [11], and it is beneficial in reducing chronic lower back pain [8], increasing oxygen uptake $[12,13]$, glucose uptake $[14,15,16]$, bone density $[17,18]$. Other forms of vibration exercise characterized by modified exercise equipment with vibrating cables [19, 20], vibrating dumbbells [21, 22, 23, 24, 25], and bespoke vibratory devices $[26,27,28,29]$ have shown to positively influence neuromuscular function suggesting this form of exercise to be potentially a suitable option for several rehabilitation programs.

In an effort to understand the physiological mechanisms involved in VE, studies have demonstrated that mechanical vibration applied to the tendon of the skeletal muscle induces an involuntary contraction termed tonic vibration reflex (TVR) [30, 31]. TVR is defined as the response of the muscle to a vibratory stress that induces an increase in the contractile force of the muscle groups involved, agonist and antagonist [32, 21]. Furthermore, while direct application of vibration has been shown to determine the TVR, consensus is lacking with regards to whole body vibration (WBV) or upper body vibration exercise modalities on their ability to elicit the TVR. Also, recent work seems to suggest this possibility [33]. Upper body vibratory exercise has been also investigated and some literature suggests various mechanisms responsible for the observed increase in neuromuscular activity determined by surface EMG $[21,28]$. 
EMG could be used to assess the effectiveness of VE modalities, however it is important to consider that during VE the EMG spectrum shows sharp peaks at the vibration frequency (SPVF) which might include a combination of signal noise and true muscle activity. Some studies considered these SPVF as motion artifacts $[34,28,35]$. Recent studies, however, have ascribed those SPVF to vibration-induced muscle activity which need to be included in the EMG analysis [36, 37, 38].

While on the upper limbs it is clear that performing resistance exercise with superimposed vibration can cause an increase in EMG activity [21, 28], it is also important to understand the fatigue aspects of such modality. In general, mechanic fatigue is characterized by a decay in force-production capability, which can be assessed by maximum voluntary contraction (MVC) test [39]. Myoelectric evidence of fatigue has been characterized by decrease in the average conduction velocity (CV) of the motor unit action potentials over the course of sustained muscle contractions [40], which can be indirectly assessed by EMG spectral compression [41]. Moreover, during fatigue, decreased excitation from Ia afferents or increased excitation threshold of the nerve fibers, have also been observed [42, 43]. A measure of motoneurons (MNs) pool excitability in different muscle conditions can be assessed by the Hoffmann reflex (H-reflex) [44].

It has been reported that direct application of tendon or muscle vibration produces a reduction in the Ia afferent transmission [45, 46]. However, the reported reflex responses following indirect vibration, e.g., WBV, are inconsistent in the literature [8]. Some studies observed an inhibition of the soleus H-reflex after WBV [47, 48, 49], in line with the results obtained after direct application of vibration on a muscle or tendon. In contrast, other studies reported increased H-reflex after WBV [50], which were suggested to be beneficial for performance improvement.

It is clear that the neuromuscular reflex response after VE remains controversial. Studies have suggested VE to produce a large degree of mechanic and myoelectric fatigue on the upper limbs while fatigue leads to decreased excitation from Ia afferents [51, 42, 43]. Furthermore, studies have reported increased H-reflex amplitude after non-exhausting VE possibly due to MN hyperexcitability [50]. The objective of this study was, therefore, to investigate the fatiguing effects of vibration on the H-reflex of the forearm muscle, e.g., Flexor Carpi Radialis (FCR), at different frequencies. We hypothesize that VE determined a higher degree of fatigue and a lower decrease in $\mathrm{H}-$ reflex amplitude in the FCR compared to the control condition, which could 
be confirmed by EMG recordings. To investigate the MN excitability, a dedicated setup was adopted that can apply the superimposition of a baseline and a vibrating load to a muscle. This setup was previously described in $\mathrm{Xu}$ et al. [52].

\section{Method}

\subsection{Subjects}

Twenty five healthy right-handed subjects (21 males and 4 females, mean age $=27 \pm 5$ years) participated in this study. No effort was made to control for training specificity. Therefore, subjects ranged from sedentary to athletic and were overall heterogeneous. The participants reported no neuromuscular disorder, no history of upper extremity surgery nor injury. Pregnant women and people with implanted pacemakers were not allowed to take part in this study because of the consequences due to possible interference with electrical stimulation. Written informed consent was obtained from every subject prior to participation in the study, and the test procedures were approved by the relevant ethical committee.

\subsection{Experimental setup}

\subsubsection{Actuator}

A dedicated electromechanical actuator was adopted for applying the superimposition of a baseline and vibrating load to a muscle [52]. Figure 1 shows a scheme of the adopted setup. The core of the setup was a threephase permanent magnet motor (MSK060C, Bosch Rexroth, Boxtel, The Netherlands), able to generate a force consisting of a constant baseline with superimposed vibration. Vibration consisted of a sinusoidal amplitude modulation of the baseline force. Figure 2 shows an example of such vibrating force. A 50-cm aluminum bar was mounted perpendicular to the motor output shaft in order to transfer the generated vibrating force via a strap to a handle, which was pulled upwards by the right arm of the subjects. The position of the bar, which was related to the flexion (angle) of the subject's wrist, was measured using a rotary encoder embedded in the motor, thus providing visual feedback supporting the subjects to maintain a stable (isometric) position. The actuator was fixed to a stiff frame of steel to improve the transmission of vibrations by reducing damping effects. A fitness bench, where the subject can lay his arm to pull the handle, was mounted above the actuator. The length of the strap connecting the handle to the bar was 


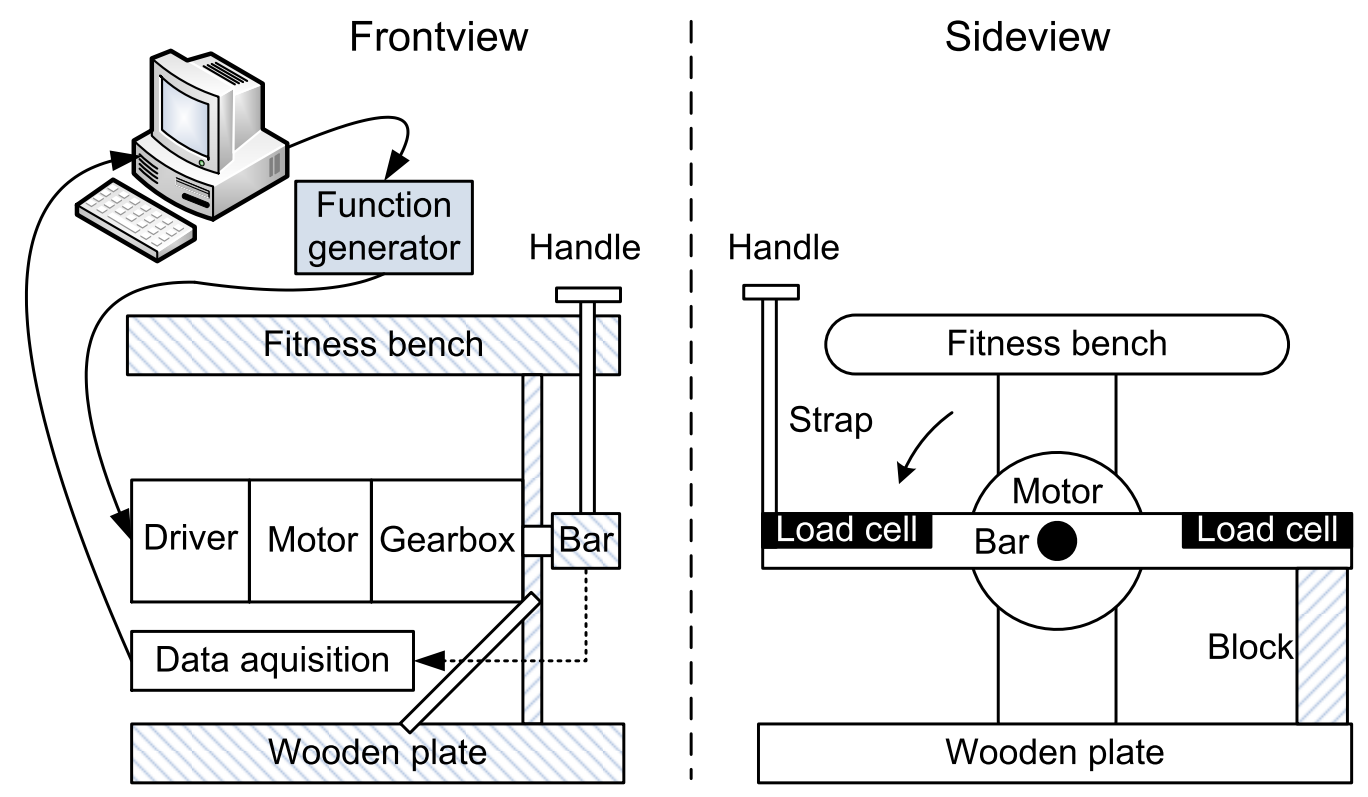

Figure 1: Scheme of the setup. The right side of the bar is blocked during the MVC test while it is free during VE trials.

adjusted to avoid any lesion of the wrist and to impose a proper level of pre-tension.

A load cell LCE-35 Kg (Omega Engineering Inc., Stamford, CT, USA) was embedded in the system to enable force measurements by using resistancebased force sensors (strain gauges) in full bridge configuration, and it was mounted on top of the bar. The input voltage to the motor drive was generated by a wave generator (PCI 5402, National Instruments, Austin, TX) connected to a PC and controlled by dedicated software implemented in LabView $^{\circledR}$ (National Instruments, Austin, TX).

\subsection{2. $E M G$ recording}

After the subjects' skin was accurately cleaned with an abrasive paste and alcohol, surface EMG was recorded from the FCR muscle by three circular $\mathrm{Ag} / \mathrm{AgCl}$ electrodes of 1-cm diameter (3M RedDot, Nadarzyn, Poland) in monopolar configuration. Two electrodes were placed on the subjects' right arm. In particular, the reference electrode was placed on the Radial Styloid, and the active electrode on the belly of the FCR muscle, as shown in Fig. 3 (a). The ground electrode was placed on top of the clavicle on the subjects' 


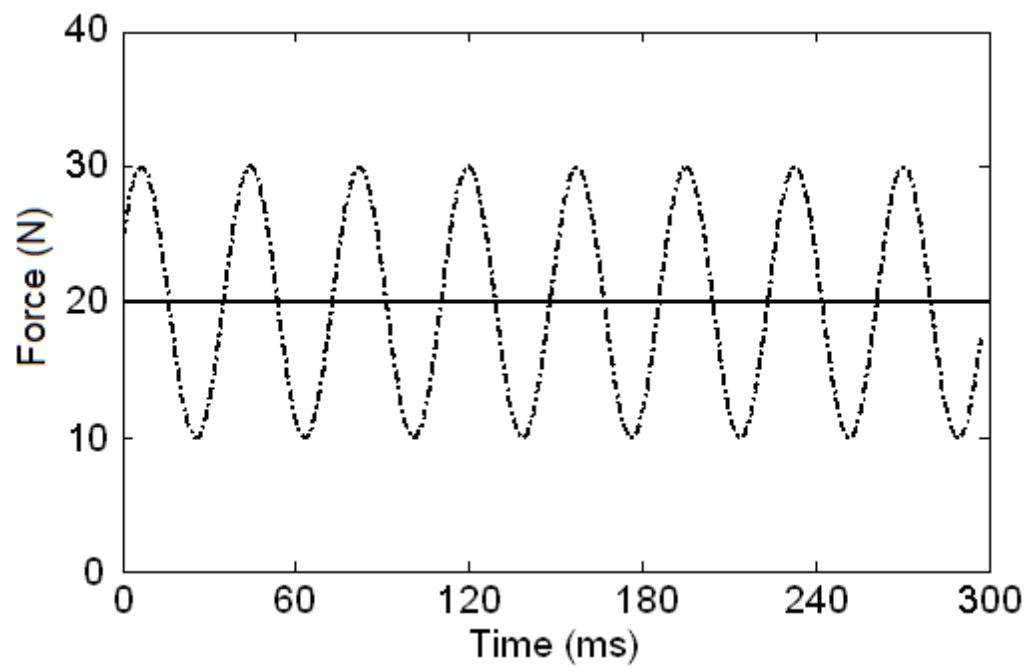

Figure 2: Example of vibrating loading function consisting of a baseline force with superimposed sinusoidal vibrating force of $10-\mathrm{N}$ amplitude.

right side. All the measured signals were amplified and digitized using a 72-channel Refa amplifier (TMS International, Enschede, The Netherlands). The sampling frequency for each channel was $2048 \mathrm{~Hz}$. Active shielding and grounding of the cables was used to minimize the powerline $(50 \mathrm{~Hz})$ interference.

\subsubsection{H-reflex elicitation}

In order to elicit the H-reflex, a stimulation electrode was used for transcutaneous stimulation of the median nerve close to the elbow, by the use of a Multi-modality electrical stimulator (Energy Light, Micromed, IT), as shown in Fig. 3, (a). By using a progressive transcutaneous electrical stimulation of the median nerve, the H-reflex tracing began to appear in the sEMG trace at low levels of stimulation as soon as the threshold of the Ia afferents was reached. When increasing stimulus intensity, the H-reflex was followed by the muscle response (M-wave) as $\alpha \mathrm{MNs}$ reached their threshold. The peakto-peak amplitude of the H-reflex increased until a maximum value, $H_{\max }$, which indicates the maximal reflex activation, and subsequently decreased. As more $\alpha \mathrm{MNs}$ were recruited, the M-wave increased until its maximum value, $M_{\max }$, which represents the activation of the entire MN pool and, therefore, the maximum muscle activation [47]. When the $\mathrm{H}$ - or M-wave 


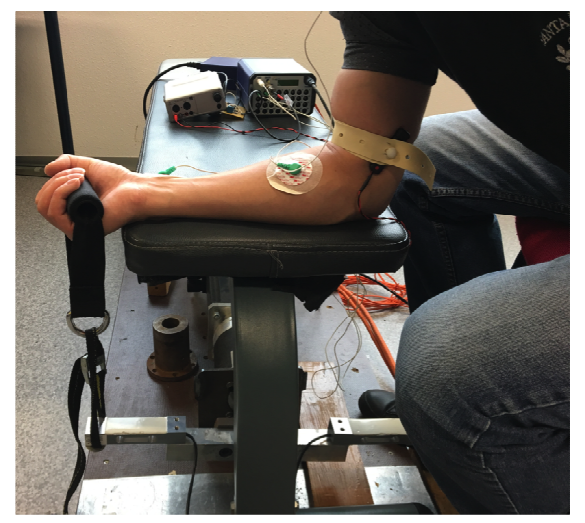

(a)

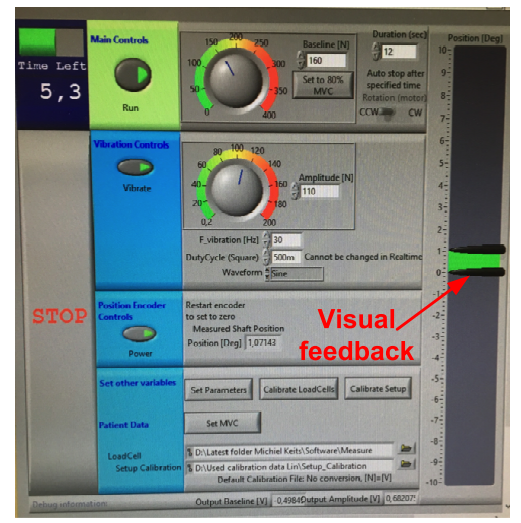

(b)

Figure 3: The measurement setup (a) together with visual feedback on the wrist position (b).

peak was reached, the corresponding value of current was established as the value of the stimulation current, $I_{H \max }$ and $I_{M \max }$, respectively. Any further increase led to a decrease in the $\mathrm{H}$ - and M-wave peaks. The stimulus intensity varied among subjects between 5 and $60 \mathrm{~mA}$ for the M-wave peak and between 1 and $20 \mathrm{~mA}$ for the $\mathrm{H}$-wave peak.

\subsection{Measurement protocol}

The whole measurement protocol is illustrated in Fig. 4. Prior to each treatment, the isometric MVC for each subject was measured by the embedded load cell. The right side of the bar of the experimental setup was blocked during the MVC measurements, as shown in Fig. 1. The subjects were asked to pull the handle with 3-s maximal isometric contraction three times, each time followed by 45 -s rest. For each 3 -s contraction, the maximum force was determined and the maximum of the three measurements was considered as the MVC. The subjects were sitting on a chair and instructed to keep their arm attached to the fitness bench with the elbow angle at about 90 degrees, which was achieved by adjusting the chair height and monitored by a professional coach. The subjects then pulled up the handle by curling the wrist without moving the arm while performing isometric contraction of the FCR. This procedure was followed for both the MVC estimation and the sustained contractions during the training protocol. During each contraction, the subjects were supported by the implemented visual feedback in order to maintain 


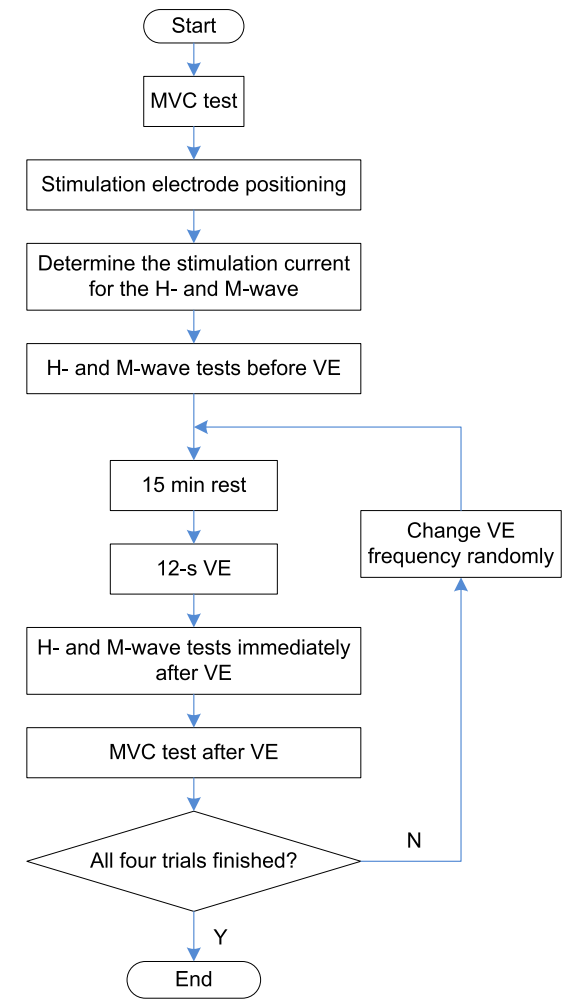

Figure 4: The schematic of the whole protocol.

the right position (Fig. 3, b). The wooden block used for the MVC test was removed during sustained contractions.

The best position for placing the stimulation electrode was determined by H-reflex screening after a transcutaneous electrical stimulation of the median nerve at $10 \%$ of the MVC. According to the H-reflex elicitation procedure, the recruitment curve of H-reflex and M-wave was then built by varying the stimulus intensity. The maximum $I_{H \max }$ and $I_{M \max }$ values were searched. The training protocol consisted of maintaining a constant muscle tension equal to $80 \%$ of the MVC with a superimposed vibration of a chosen amplitude equal to $70 \%$ of the baseline tension.

The experimental conditions were characterized by four sustained (12-s) isometric contractions of the right FCR muscle with no vibration (control), and with vibration at 15,30 , and $45 \mathrm{~Hz}$. A randomized cross-over design was used. Each condition was followed by a 15-min resting period. This was experimentally proven to permit full MVC recovery. To this end, different 
recovery intervals were tested incrementally: 5, 10, and 15 min. Eventually, 15 min were found sufficient for full recovery of the muscle performance, since the subjects could produce the same level of MVC as prior to the fatiguing task. In addition, this choice was in line with literature [51], in which a recovery period of 12 min was adopted due to slightly lower vibration amplitude ( $60 \%$ of the baseline force). Before and after each trial, the FCR MVC, together with the $\mathrm{H}$ - and $\mathrm{M}$-wave, were measured following the same protocol as before training. Ten repeated rectangular pulses of $1000 \mu$ s duration each at a triggering frequency of $0.5 \mathrm{~Hz}$ were delivered to elicit the H-reflex response for determining the maximal $\mathrm{H}$ - and $\mathrm{M}$-waves before and after each trial.

\subsection{Signal analysis \\ 2.4.1. Mechanical fatigue}

The MVC test was performed before and after each trial in order to assess mechanical fatigue. The MVC for each subject was measured by the embedded load cell. First, the shaft of the motor was blocked in order to perform an isometric contraction and to measure by the embedded load cell the produced force. Mechanical fatigue was then assessed by measuring the absolute change in MVC after each trial as compared to before exercise $(\triangle M V C)$.

\subsubsection{Myoelectric fatigue}

During the four sustained isometric contractions, the sEMG was recorded from the FCR muscle. To provide an exhaustive evaluation of the fatigue effect introduced by VE, myoelectric fatigue was assessed by estimating the mean frequency (MF). According to recent works [36, 37, 38], SPVF during VE consist mainly of vibration-induced muscle activity; therefore the frequency spectrum in a bandwidth of $20-450 \mathrm{~Hz}$ [53] was included in the EMG analysis without additional filtering. The MF was then estimated by time-frequency analysis of the bipolar EMG signal. A sliding window of 1-s with no overlap was adopted to compute the Short-time Fourier Transform. The MF was then estimated as the first statistical moment of the obtained amplitude spectrum. For each segment, the MF was obtained as:

$$
M F=\frac{\sum_{f=20}^{450} f \cdot S_{f}}{\sum_{f=20}^{450} S_{f}},
$$

where $f$ is the frequency $[\mathrm{Hz}]$ and $S_{n, f}$ is the amplitude spectrum at frequency $f$. A linear regression was then applied to the values obtained from all the 
windows and the angular coefficient of the regression line used as indicator of myoelectric muscle fatigue $[27,51]$.

\subsubsection{H-reflex}

According to the H-reflex elicitation procedure described in section 2.2, ten $\mathrm{H}$ - and M-waves were recorded before and after VE for each trial by stimulating the median nerve. For each recording the ten $\mathrm{H}-$ and $\mathrm{M}$-waves were first averaged. The peak-to-peak amplitude of the averaged $\mathrm{H}-$ and M-wave was then calculated. In general, the peak-to-peak amplitude of the $\mathrm{H}$-wave, $H_{\max }$, is an indirect estimate of the number of MNs that are able to activate in a given state while the peak-to-peak amplitude of the Mwave, $M_{\max }$, represents the activation of the entire MN pool; therefore, the calculated $H_{\max }$ was normalized with respect to the $M_{\max }$, indicating the fraction of the entire MN pool that can be recruited [54].

The normalized $H_{\max }$ was estimated both before and after the exercise at different vibration frequencies $(0,15,30$, and $45 \mathrm{~Hz})$. The relative decrease in the normalized $H_{\max }$ after each exercise was then calculated as indication of H-reflex suppression, as given by

$$
\Delta H=\frac{H_{\text {max }, a \text { fter }}-H_{\text {max }, \text { before }}}{H_{\text {max }, \text { before }}} \times 100 \% .
$$

\subsection{Statistical analysis}

The parameters investigated in our statistical analysis are the H-reflex suppression, myoelectric fatigue, and mechanical fatigue. H-reflex suppression was estimated as the decrease in H-reflex amplitude after each trial. Myoelectric fatigue was estimated as the slope of the regression line to the time evolution of the MF, as assessed during each trial. Mechanical fatigue was estimated as the drop in MVC measured after each trial. Subjects were excluded from the H-reflex analysis (3 subjects) based on one criterium: signal-to-noise ratio $<18 \mathrm{~dB}$ for the H-reflex response. This threshold value was based on visual inspection. According to the one-sample KolmogorovSmirnov test [55], all data were Gaussian distributed ( $p>0.05)$. However, for each subject, the data recorded with different test conditions, i.e., frequencies, are not independent. As a consequence, the one tailed Wilcoxon signed rank test was adopted to establish the significance level ( $p$ value) in the mean values between control (no vibration) and each vibration frequency. For all the analyzes, $\alpha$ was set at 0.05 . 


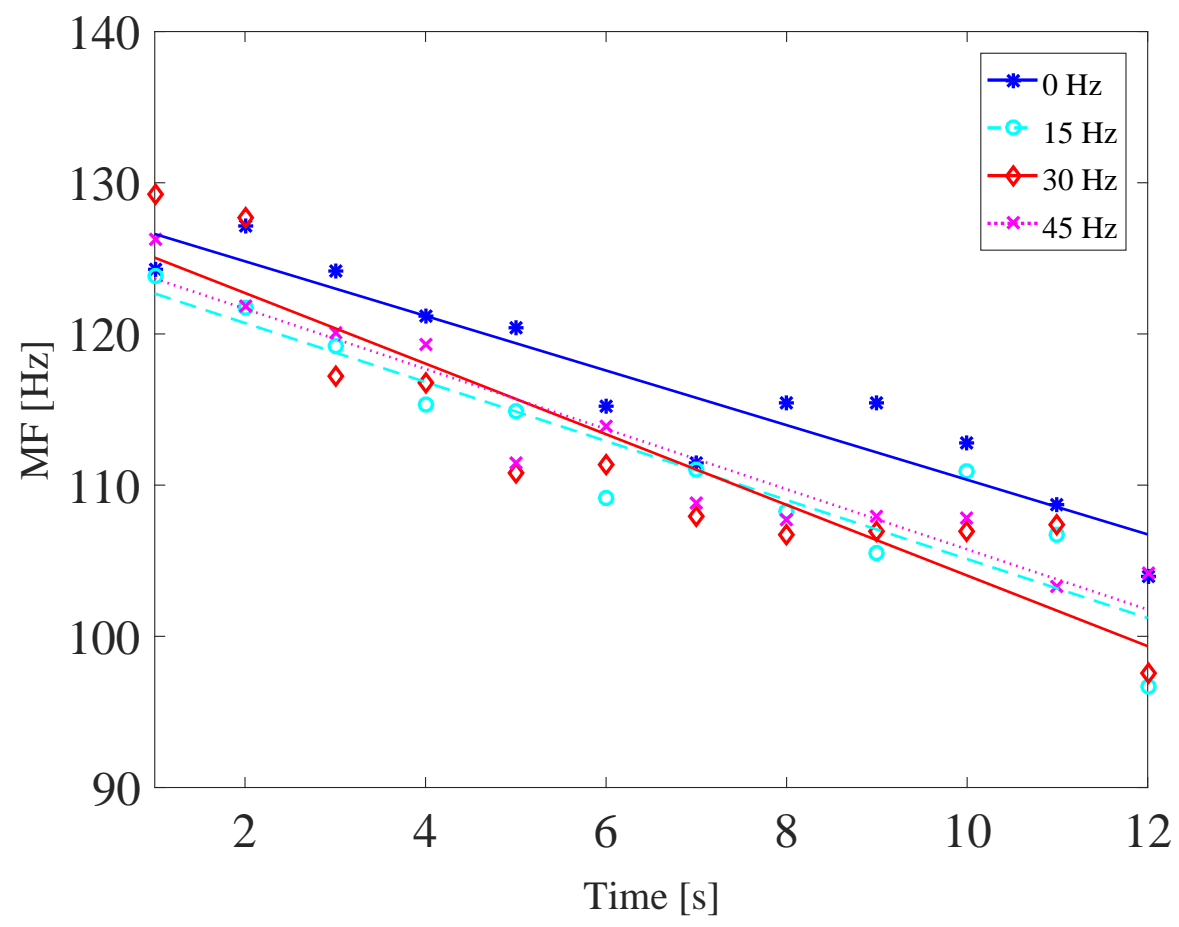

Figure 5: Example of MF estimates for varying vibration frequencies from 0 (control with no vibration) to $45 \mathrm{~Hz}$.

\section{Results}

\subsection{Myoelectric and mechanical fatigue indicators}

Figure 5 shows the MF time evolution for a subject. In all the measurements, the MF evolution over time showed a trend that was well approximated by a linear model. The average $\mathrm{R}^{2}$ (determination coefficient) value of the regression lines over the full dataset was $0.83 \pm 0.11$. Myoelectric fatigue was therefore estimated as the (negative) angular coefficient of the regression lines. Figure 6 shows the averaged MF slope over all the subjects for each corresponding training at $0,15,30$, and $45 \mathrm{~Hz}$ in terms of mean \pm standard deviation. Figure 7 shows the MVC decay over all the subjects for each corresponding training at $0,15,30$, and $45 \mathrm{~Hz}$ in terms of mean \pm standard deviation. The estimates in Fig. 6 and Fig. 7 indicate $30 \mathrm{~Hz}$ as the most fatiguing exercise, according to both the MF and the MVC assessment. The results of our statistical analysis on MF and MVC reveal the separation 


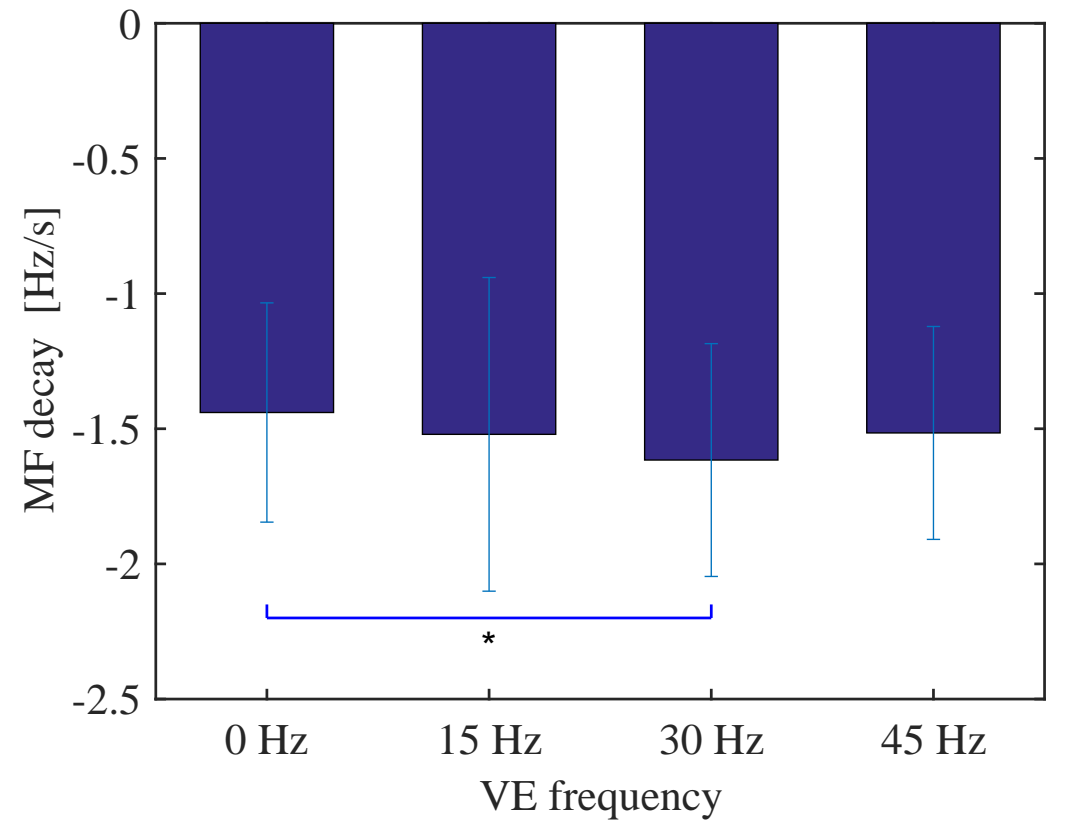

Figure 6: MF decay estimates for varying vibration frequencies from 0 (control with no vibration) to $45 \mathrm{~Hz}$ in terms of mean \pm standard deviation. The asterisk $\left(^{*}\right)$ indicates the significant difference $(\mathrm{p}<0.05)$ between $0 \mathrm{~Hz}$ and $30 \mathrm{~Hz}$.

of different groups to be significant only between $0 \mathrm{~Hz}$ and $30 \mathrm{~Hz}(\mathrm{p}<0.05)$.

\subsection{H-reflex suppression}

Figure 8 represents an example of the ten H-reflex responses recorded for one subject after VE at $30 \mathrm{~Hz}$. Table 1 reports the normalized H-reflex values in terms of mean \pm standard deviation, while Fig. 9 depicts the $\Delta H$ in terms of mean \pm standard deviation. These results show a decay in the normalized H-reflex amplitude after fatiguing exercise at all the frequencies as compared to before exercise. In addition, as compared to control condition $(0 \mathrm{~Hz})$, the H-reflex amplitude decreases more at $15 \mathrm{~Hz}$ than at 30 and $45 \mathrm{~Hz}$. The lowest H-reflex amplitude suppression is observed at $30 \mathrm{~Hz}$. The results of our statistical analysis on $\Delta H$ reveals the separation of different groups to be significant only between $0 \mathrm{~Hz}$ and $30 \mathrm{~Hz}(\mathrm{p}<0.05)$. 


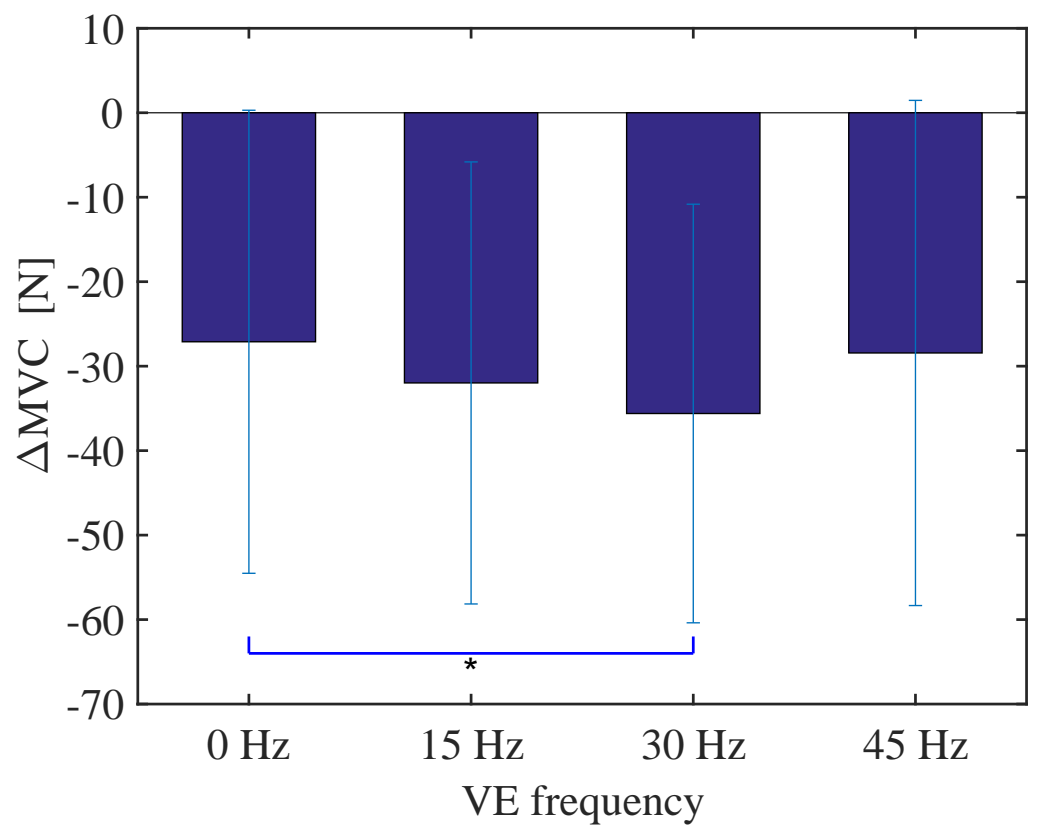

Figure 7: $\triangle M V C$ estimates for varying vibration frequencies from 0 (control with no vibration) to $45 \mathrm{~Hz}$ in terms of mean \pm standard deviation. The asterisk $(*)$ indicates the significant difference $(\mathrm{p}<0.05)$ between $0 \mathrm{~Hz}$ and $30 \mathrm{~Hz}$.

\section{Discussion}

In line with previous research [51], as indicated by MVC and MF indicators, our results show that vibration produced a larger degree of mechanical and myoelectric fatigue as compared to control condition $(0 \mathrm{~Hz})$, showing 30 $\mathrm{Hz}$ vibration as the most fatiguing exercise. It should be noticed that SPVF are indeed observed in our EMG measurements. However, since many recent studies suggest those SPVF to be vibration-induced muscle activity, they are not excluded in our MF calculation [36, 37, 38].

Several studies $[47,56,57]$ have investigated the changes in MN pool excitability after the superimposition of vibration, showing how the H-reflex amplitude is suppressed after vibration-induced fatigue. Our results show that after fatiguing exercise the H-reflex amplitude decreased by $28 \%$ compared to before exercise; this result is in line with literature [58]. When applying vibration a lower suppression of the H-reflex amplitude was observed as compared to control condition $(0 \mathrm{~Hz})$, with the smallest suppression $(20 \%)$ 


\begin{tabular}{lll}
\hline$f(\mathbf{H z})$ & $H_{\text {max }, \text { before }}$ & $H_{\text {max }, \text { after }}$ \\
\hline 0 & $0.1666 \pm 0.1073$ & $0.1185 \pm 0.0921$ \\
15 & $/$ & $0.1122 \pm 0.0713$ \\
30 & $/$ & $0.1285 \pm 0.0822$ \\
45 & $/$ & $0.1208 \pm 0.0859$ \\
\hline
\end{tabular}

Table 1: Normalized H-reflex amplitude before and after VE at different frequencies.

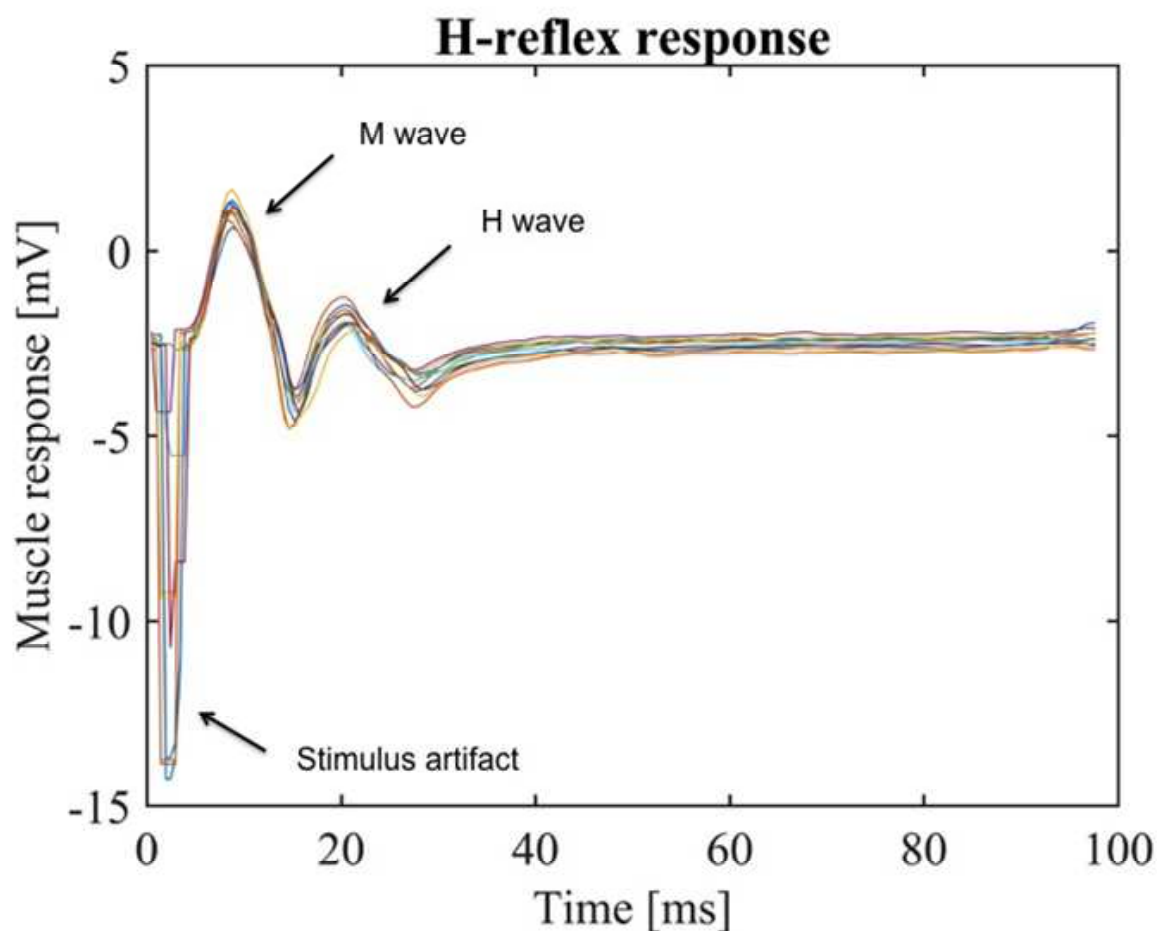

Figure 8: Example of ten H-reflex responses recorded for one subject with the stimulation current $I_{H \max }$.

at $30 \mathrm{~Hz}$.

The observed H-reflex amplitude suppression reflected in the reduction of the FCR MN pool excitability and a change in the synaptic efficacy, which can be explained by reflex inhibition from group III and IV afferents [59]. The major finding of our study is that the H-reflex amplitude decreased less compared to control condition $(0 \mathrm{~Hz})$ when vibration is applied. Therefore, the VE protocol used in this study seems associated with an enhancement in the FCR MN pool excitability, possibly due to TVR [30,31], reflected in a 


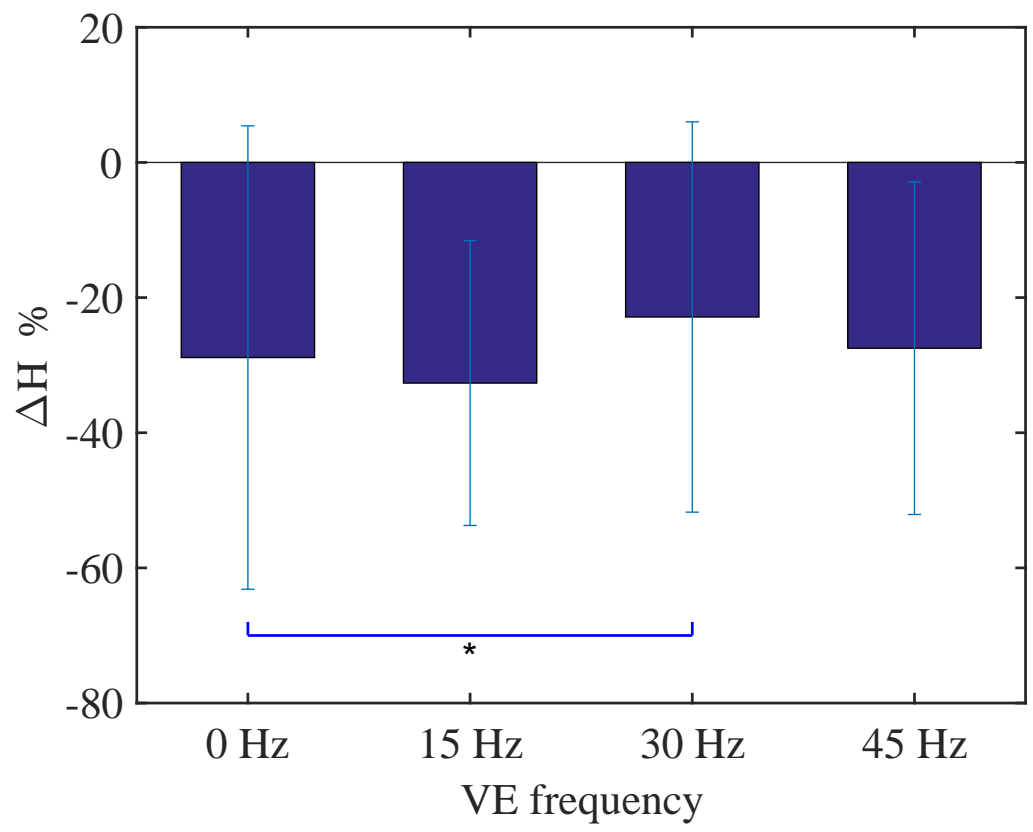

Figure 9: $\Delta H$ estimates for varying vibration frequencies from 0 (control with no vibration) to $45 \mathrm{~Hz}$ in terms of mean \pm standard deviation. The asterisk $\left(^{*}\right)$ indicates the significant difference $(\mathrm{p}<0.05)$ between $0 \mathrm{~Hz}$ and $30 \mathrm{~Hz}$.

lower suppression of the H-reflex amplitude as compared to control condition $(0 \mathrm{~Hz})$. This phenomenon was not evident at $15 \mathrm{~Hz}$, possibly because this frequency is not suitable to stimulate effectively TVR and MN excitability.

In the light of these results, we may hypothesize that VE, especially at $30 \mathrm{~Hz}$, enhances mechanical and myoelectric fatigue, which are reflected in MVC decay and EMG spectral compression, respectively, while limiting the reduction in MN excitability, which is reflected in a smaller H-reflex suppression. At this stage, considering recent work on whole body vibration [60], we should not exclude a central influence, as the reduction in force generating capacity observed may occur not only because of peripheral changes at the muscular level, but also because the central nervous system fails to drive the MNs adequately [61]. This could suggest $30 \mathrm{~Hz}$ to provide effective enhancement of the peripheral component as compared to the central component and neural drive decay. Dedicated studies on the ability to achieve full muscle activation, e.g., via twitch interpolation techniques [62], may elucidate on the central and peripheral fatigue involvement as well as on the contribution 
of spinal mechanisms as attempted with WBV.

As Amstrong et al. [47] reported, the H-reflex response to a vibratory stress is highly variable and may be dependent on muscle fiber type differences. Moreover, the post-activation of the H-reflex response may be dependent on the nature of the stimulus (e.g., frequency and intensity) as well as on the complex interaction between the muscle fiber fatigue characteristics and the nature of the fatiguing protocol. Although our dedicated protocol aims at applying superimposed VE on the FCR muscle, it is not possible to eliminate the involvement of other muscle groups of the forearm. As a result, crosstalk from other muscle groups of the forearm may be present in the EMG recordings.

It should also be noted that the aim of the present study is to investigate vibration-induced effects on muscle fatigue and H-reflex. We mainly focus on the different effects between vibration and control condition (no vibration). Moreover, for each subject, the data recorded with and without vibration are not independent. As a consequence, Wilcoxon test between control and each vibration frequency is adopted for the statistical analysis. However, the effects between different vibration frequencies might also be of interest for other purposes. In this case, a multi-comparison should be performed for the statistical analysis by using repeated measures of ANOVA and post-hoc test.

\section{Conclusion}

In the present study, the effects of vibration-induced fatigue were investigated by analysis of the H-reflex response and myolectric and mechanical fatigue indicators. Our results demonstrate a lower fatigue-induced reflex inhibition of the FCR MN pool after VE. This effect was especially evident at $30 \mathrm{~Hz}$. In parallel, $30 \mathrm{~Hz}$ caused the largest myoelectric (EMG spectral compression) and mechanical (MVC decay) evidence of acute neuromuscular impairment, resulting in the most fatiguing frequency for training. This suggests VE, especially at $30 \mathrm{~Hz}$, to enhance mechanical and myoelectric fatigue while limiting the reduction in MN excitability. These results highlight the need for further investigation of spinal reflex mechanisms involvement as well as their complex interaction with fatigue. They also indicate that in the FCR muscle, undergoing VE in isometric conditions, peripheral inhibitions may be accounted for the MVC reduction with our protocol, but central components should not be excluded and should be verified experimentally. This feature may turn out to be particularly suitable in rehabilitation programs targeting 
the arm following immobilisation periods to restore muscle form and function rapidly. In general, our results may be used when personalizing VE training protocols involving the FCR.

Further studies are needed to determine safe and effective protocols of VE using our similar modalities to exercise upper body muscles as well as to understand the acute and chronic implications on the musculoskeletal system.

\section{Conflict of interest}

The authors have no financial affiliation (including research funding) or involvement with any commercial organization that has a direct financial interest in any matter included in this manuscript.

\section{References}

[1] W. J. Kraemer, et al., American college of sports medicine position stand. progression models in resistance training for healthy adults., Med Sci Sport Exe 34 (2002) 364-380.

[2] A. D. Faigenbaum, W. J. Kraemer, C. J. Blimkie, I. Jeffreys, L. J. Micheli, M. Nitka, T. W. Rowland, Youth resistance training: updated position statement paper from the national strength and conditioning association, The Journal of Strength \& Conditioning Research 23 (2009) S60-S79.

[3] G. J. Wilson, R. U. Newton, A. J. Murphy, B. J. Humphries, The optimal training load for the development of dynamic athletic performance., Med Sci Sport Exe 25 (1993) 1279-1286.

[4] T. Hortobágyi, N. A. Maffiuletti, Neural adaptations to electrical stimulation strength training, European journal of applied physiology 111 (2011) 2439-2449.

[5] B. R. Scott, J. P. Loenneke, K. M. Slattery, B. J. Dascombe, Exercise with blood flow restriction: an updated evidence-based approach for enhanced muscular development, Sports medicine 45 (2015) 313-325.

[6] M. Cardinale, J. Wakeling, Whole body vibration exercise: are vibrations good for you?, British journal of sports medicine 39 (2005) $585-589$. 
[7] M. Cardinale, C. Bosco, The use of vibration as an exercise intervention, Exercise and sport sciences reviews 31 (2003) 3-7.

[8] J. Rittweger, Vibration as an exercise modality: how it may work, and what its potential might be, Eur J Appl Physiol 108 (2010) 877-904.

[9] Y. Osawa, Y. Oguma, N. Ishii, The effects of whole-body vibration on muscle strength and power: a meta-analysis, J Musculoskelet Neuronal Interact 13 (2013) 380-390.

[10] R. Ritzmann, A. Kramer, S. Bernhardt, A. Gollhofer, Whole body vibration training-improving balance control and muscle endurance, PloS one 9 (2014).

[11] C. Delecluse, M. Roelants, et al., Strength increase after whole-body vibration compared with resistance training, Med Sci Sport Exe 35 (2003) 1033-1041.

[12] E. Yamada, T. Kusaka, et al., Vastus lateralis oxygenation and blood volume measured by near-infrared spectroscopy during whole body vibration, Clin Physiol Funct Imaging 25 (2005) 203-208.

[13] M. Cardinale, M. Ferrari, V. Quaresima, Gastrocnemius medialis and vastus lateralis oxygenation during whole-body vibration exercise, Med Sci Sport Exe 39 (2007) 694-700.

[14] P. Lucidi, G. Murdolo, et al., Meal intake similarly reduces circulating concentrations of octanoyl and total ghrelin in humans, Journal of endocrinological investigation 27 (2004) RC15-RC15.

[15] C. Di Loreto, A. Ranchelli, P. Lucidi, G. Murdolo, N. Parlanti, A. De Cicco, O. Tsarpela, G. Annino, C. Bosco, F. Santeusanio, et al., Effects of whole-body vibration exercise on the endocrine system of healthy men, Journal of endocrinological investigation (2004) 323-327.

[16] K. Baum, T. Votteler, J. Schiab, Efficiency of vibration exercise for glycemic control in type 2 diabetes patients, Int J Med Sci 4 (2007) 159.

[17] C. Rubin, A. S. Turner, et al., Anabolism: Low mechanical signals strengthen long bones, Nature 412 (2001) 603-604. 
[18] S. M. Verschueren, M. Roelants, et al., Effect of 6-month whole body vibration training on hip density, muscle strength, and postural control in postmenopausal women: A randomized controlled pilot study, Journal of bone and mineral research 19 (2004) 352-359.

[19] V. Issurin, D. Liebermann, G. Tenenbaum, Effect of vibratory stimulation training on maximal force and flexibility, Journal of sports sciences 12 (1994) 561-566.

[20] V. Issurin, G. Tenenbaum, Acute and residual effects of vibratory stimulation on explosive strength in elite and amateur athletes, Journal of sports sciences 17 (1999) 177-182.

[21] C. Bosco, M. Cardinale, O. Tsarpela, Influence of vibration on mechanical power and electromyogram activity in human arm flexor muscles, Eur J Appl Physiol 79 (1999) 306-311.

[22] D. Cochrane, S. Stannard, A. Walmsely, E. Firth, The acute effect of vibration exercise on concentric muscular characteristics, Journal of science and medicine in sport 11 (2008) 527-534.

[23] D. J. Cochrane, E. J. Hawke, Effects of acute upper-body vibration on strength and power variables in climbers., The Journal of Strength \& Conditioning Research 21 (2007) 527-531.

[24] J. M. McBride, J. P. Porcari, M. D. Scheunke, Effect of vibration during fatiguing resistance exercise on subsequent muscle activity during maximal voluntary isometric contractions., The Journal of Strength \& Conditioning Research 18 (2004) 777-781.

[25] D. Fowler, M. Tok, M. Colakoğlu, F. Bademkiran, Z. Colakoğlu, Exercise with vibration dumb-bell enhances neuromuscular excitability measured using tms., The Journal of sports medicine and physical fitness 50 (2010) 336-342.

[26] M. Mischi, C. Rabotti, M. Cardinale, Analysis of muscle fatigue induced by isometric vibration exercise at varying frequencies, in: Engineering in Medicine and Biology Society (EMBC), 2012 Annual International Conference of the IEEE, IEEE, pp. 6463-6466. 
[27] M. Mischi, C. Rabotti, M. Cardinale, Electromyographic assessment of muscle fatigue during isometric vibration training at varying frequencies, in: Engineering in Medicine and Biology Society (EMBC), 2010 Annual International Conference of the IEEE, IEEE, pp. 2338-2341.

[28] M. Mischi, M. Cardinale, The effects of a $28-\mathrm{Hz}$ vibration on arm muscle activity during isometric exercise, Med Sci Sport Exe 41 (2009) 645-652.

[29] A. N. Pujari, R. D. Neilson, S. S. Aphale, M. Cardinale, Upper limb vibration prototype with sports and rehabilitation applications: development, evaluation and preliminary study, Healthcare Technology Letters 4 (2017) 44-49.

[30] G. Eklund, K.-E. Hagbarth, Normal variability of tonic vibration reflexes in man, Exp Neurology 16 (1966) 80-92.

[31] K.-E. Hagbarth, G. Eklund, Tonic vibration reflexes (tvr) in spasticity, Brain research 2 (1966) 201-203.

[32] L. Bongiovanni, K. Hagbarth, Tonic vibration reflexes elicited during fatigue from maximal voluntary contractions in man., The Journal of physiology 423 (1990) 1-14.

[33] L. N. Zaidell, K. N. Mileva, D. P. Sumners, J. L. Bowtell, Experimental evidence of the tonic vibration reflex during whole-body vibration of the loaded and unloaded leg, PloS one 8 (2013) e85247.

[34] A. F. Abercromby, W. E. Amonette, C. S. Layne, B. K. Mcfarlin, M. R. Hinman, W. H. Paloski, Variation in neuromuscular responses during acute whole-body vibration exercise., Med Sci Sports Exerc 39 (2007) $1642-1650$.

[35] B. J. Martin, H.-S. Park, Analysis of the tonic vibration reflex: influence of vibration variables on motor unit synchronization and fatigue, European journal of applied physiology and occupational physiology 75 (1997) 504-511.

[36] A. Fratini, M. Cesarelli, P. Bifulco, M. Romano, Relevance of motion artifact in electromyography recordings during vibration treatment, Journal of Electromyography and Kinesiology 19 (2009) 710-718. 
[37] L. Xu, C. Rabotti, M. Mischi, On the nature of the electromyographic signals recorded during vibration exercise, European journal of applied physiology (2015) 1-12.

[38] R. Ritzmann, A. Kramer, M. Gruber, A. Gollhofer, W. Taube, Emg activity during whole body vibration: motion artifacts or stretch reflexes?, European journal of applied physiology 110 (2010) 143-151.

[39] D. Jones, B. Bigland-Ritchie, R. Edwards, Excitation frequency and muscle fatigue: mechanical responses during voluntary and stimulated contractions, Exp Neur 64 (1979) 401-413.

[40] R. Merletti, M. Knaflitz, C. J. De Luca, Myoelectric manifestations of fatigue in voluntary and electrically elicited contractions, J Appl Physiol 69 (1990) 1810-1820.

[41] M. Lowery, P. Nolan, M. Omalley, Electromyogram median frequency, spectral compression and muscle fibre conduction velocity during sustained sub-maximal contraction of the brachioradialis muscle, J Electromyo Kine 12 (2002) 111-118.

[42] G. Macefield, K.-E. Hagbarth, et al., Decline in spindle support to alphamotoneurones during sustained voluntary contractions., J Physiol 440 (1991) 497-512.

[43] D. Neyroud, A. Vallotton, G. Y. Millet, B. Kayser, N. Place, The effect of muscle fatigue on stimulus intensity requirements for central and peripheral fatigue quantification, European journal of applied physiology 114 (2014) 205-215.

[44] M. Hugon, Methodology of the hoffmann reflex in man, Devel Electromyo Clin Neurophy 3 (1973) 277-293.

[45] C. S. Arcangel, R. Johnston, B. Bishop, The achilles tendon reflex and the h-response during and after tendon vibration, Physical therapy 51 (1971) 889-905.

[46] A. Van Boxtel, Differential effects of low-frequency depression, vibration-induced inhibition, and posttetanic potentiation on h-reflexes and tendon jerks in the human soleus muscle, Journal of Neurophysiology 55 (1986) 551-568. 
[47] W. J. Armstrong, H. N. Nestle, D. C. Grinnell, L. D. Cole, E. L. Van Gilder, G. S. Warren, E. A. Capizzi, The acute effect of wholebody vibration on the hoffmann reflex, The Journal of Strength \& Conditioning Research 22 (2008) 471-476.

[48] D. G. Sayenko, K. Masani, M. Alizadeh-Meghrazi, M. R. Popovic, B. C. Craven, Acute effects of whole body vibration during passive standing on soleus h-reflex in subjects with and without spinal cord injury, Neuroscience letters 482 (2010) 66-70.

[49] R. Ritzmann, A. Kramer, A. Gollhofer, W. Taube, The effect of whole body vibration on the h-reflex, the stretch reflex, and the short-latency response during hopping, Scandinavian journal of medicine \& science in sports 23 (2013) 331-339.

[50] J. Rittweger, M. Mutschelknauss, D. Felsenberg, Acute changes in neuromuscular excitability after exhaustive whole body vibration exercise as compared to exhaustion by squatting exercise, Clinical physiology and functional imaging 23 (2003) 81-86.

[51] L. Xu, C. Rabotti, M. Mischi, Analysis of vibration exercise at varying frequencies by different fatigue estimators, IEEE Transactions on Neural Systems and Rehabilitation Engineering 24 (2016) 1284-1293.

[52] L. Xu, C. Rabotti, M. Mischi, Novel vibration-exercise instrument with dedicated adaptive filtering for electromyographic investigation of neuromuscular activation, Neural Systems and Rehabilitation Engineering, IEEE Transactions on 21 (2013) 275-282.

[53] E. A. Clancy, E. L. Morin, R. Merletti, Sampling, noise-reduction and amplitude estimation issues in surface electromyography, Journal of electromyography and kinesiology 12 (2002) 1-16.

[54] R. M. Palmieri, C. D. Ingersoll, M. A. Hoffman, The hoffmann reflex: methodologic considerations and applications for use in sports medicine and athletic training research, J Athl Train 39 (2004) 268-277.

[55] R. H. Lopes, Kolmogorov-smirnov test, in: International Encyclopedia of Statistical Science, Springer, 2011, pp. 718-720. 
[56] J. M. McBride, J. L. Nuzzo, A. M. Dayne, M. A. Israetel, D. C. Nieman, N. T. Triplett, Effect of an acute bout of whole body vibration exercise on muscle force output and motor neuron excitability, The Journal of Strength \& Conditioning Research 24 (2010) 184-189.

[57] K. Kipp, S. T. Johnson, J. R. Doeringer, M. A. Hoffman, Spinal reflex excitability and homosynaptic depression after a bout of whole-body vibration, Muscle \& nerve 43 (2011) 259-262.

[58] J. Duchateau, K. Hainaut, Behaviour of short and long latency reflexes in fatigued human muscles., The Journal of Physiology 471 (1993) 787799 .

[59] P. Rudomin, R. F. Schmidt, Presynaptic inhibition in the vertebrate spinal cord revisited, Experimental Brain Research 129 (1999) 1-37.

[60] A. Krause, A. Gollhofer, K. Freyler, L. Jablonka, R. Ritzmann, Acute corticospinal and spinal modulation after whole body vibration., Journal of musculoskeletal \& neuronal interactions 16 (2016) 327-338.

[61] S. Gandevia, Spinal and supraspinal factors in human muscle fatigue, Physiological reviews 81 (2001) 1725-1789.

[62] P. Merton, Voluntary strength and fatigue, The Journal of physiology 123 (1954) 553-564. 